Applied Mathematical Sciences, Vol. 7, 2013, no. 125, 6209 - 6216

HIKARI Ltd, www.m-hikari.com

http://dx.doi.org/10.12988/ams.2013.39492

\title{
The Asymptotic Expansion of a Generalised Mathieu Series
}

\author{
R. B. Paris
}

School of Engineering, Computing and Applied Mathematics University of Abertay Dundee, Dundee DD1 1HG, UK

Copyright (C) 2013 R. B. Paris. This is an open access article distributed under the Creative Commons Attribution License, which permits unrestricted use, distribution, and reproduction in any medium, provided the original work is properly cited.

\begin{abstract}
We obtain the asymptotic expansion of a generalised Mathieu series and its alternating variant for large complex values of the variable by means of a Mellin transform approach. Numerical examples are presented to demonstrate the accuracy of these expansions.
\end{abstract}

Mathematics Subject Classification: 30E15, 30E20, 34E05

Keywords: Asymptotic expansions, generalised Mathieu series, Mellin transform method

\section{Introduction}

The functional series

$$
S_{\mu}(a)=\sum_{n=1}^{\infty} \frac{n}{\left(n^{2}+a^{2}\right)^{\mu}} \quad(\mu>1)
$$

in the case $\mu=2$ was introduced by Mathieu in his 1890 book [5] dealing with the elasticity of solid bodies. Considerable effort has been devoted to the determination of upper and lower bounds for this series when the parameter $a>0$; see [10] and the references therein.

Various integral representations have been obtained for the series $S_{\mu}(a)$, together with its alternating variant

$$
\tilde{S}_{\mu}(a)=\sum_{n=1}^{\infty} \frac{(-1)^{n-1} n}{\left(n^{2}+a^{2}\right)^{\mu}} \quad\left(\mu>\frac{1}{2}\right) .
$$


We have

$$
\left.\begin{array}{l}
S_{\mu}(a) \\
\tilde{S}_{\mu}(a)
\end{array}\right\}=\frac{\pi^{\frac{1}{2}}(2 a)^{\frac{3}{2}-\mu}}{2 \Gamma(\mu)} \int_{0}^{\infty} \frac{t^{\mu-\frac{1}{2}}}{e^{t} \mp 1} J_{\mu-\frac{3}{2}}(a t) d t \quad(a>0)
$$

where $J_{\nu}(x)$ denotes the Bessel function of the first kind of order $\nu$ and the upper or lower sign corresponds to $S_{\mu}(a)$ or $\tilde{S}_{\mu}(a)$, respectively. When $\mu=2$, these reduce to the integrals

$$
S_{2}(a)=\frac{1}{2 a} \int_{0}^{\infty} \frac{t \sin (a t)}{e^{t}-1} d t, \quad \tilde{S}_{2}(a)=\frac{1}{2 a} \int_{0}^{\infty} \frac{t \sin (a t)}{e^{t}+1} d t
$$

for $a>0$, given by Emersleben [2] and Pogány et al. [10], respectively. Other integral representations involving the hyperbolic functions have recently been derived in [6].

Our objective in this paper is to derive the asymptotic expansion of the more general functional series ${ }^{1}$

$$
\left.\begin{array}{l}
S_{\mu, \gamma}(a ; b) \\
\tilde{S}_{\mu, \gamma}(a ; b)
\end{array}\right\}=\sum_{n=1}^{\infty} \frac{( \pm 1)^{n-1} n^{\gamma}}{\left(n^{2}+a^{2}\right)^{\mu}} \exp \left[\frac{-a^{2} b}{n^{2}+a^{2}}\right] \quad(\mu>0, \gamma>-1, b \in \mathbf{R})
$$

for large complex $a$, where it is supposed for absolute convergence that $\delta:=$ $2 \mu-\gamma>1$. Since $S_{\mu, \gamma}(a ; b)$ and $\tilde{S}_{\mu, \gamma}(a ; b)$ are both even in $a$ it is sufficient to consider $|\arg a| \leq \frac{1}{2} \pi$. The series reduce to $S_{2}(a)$ and $\tilde{S}_{2}(a)$ when $\gamma=1, b=0$ and $\mu=2$. The presence of the exponential $e_{n}:=\exp \left[-a^{2} b /\left(n^{2}+a^{2}\right)\right]$ acts as a perturbing factor which, although it does not affect the rate of convergence of the series ( since $e_{n} \rightarrow 1$ as $n \rightarrow \infty$ ), can disturb the extreme cancellation that takes place in the alternating series and so significantly modify its large- $a$ growth. The effect of similar perturbing factors on the negative exponential series for $e^{-x}$ has been discussed in [8], where it was established that the growth for $x \rightarrow+\infty$ can be quite different from the unperturbed case. The asymptotic expansions of $S_{\mu}(a)$ and $\tilde{S}_{\mu}(a)$ as $a \rightarrow+\infty$ have been given in $[1,10]$. Asymptotic expansions of $S_{\mu, \gamma}(a ; 0)$ and $\tilde{S}_{\mu, \gamma}(a ; 0)$ for $a \rightarrow+\infty$ have been considered in [12] using a Mellin transform approach. We adopt a similar approach since it easily enables us to deal with complex $a$; for a description of this method see, for example, [9, Section 4.1.1].

In the application of the Mellin transform method to the series $S_{\mu, \gamma}(a ; b)$ and $\tilde{S}_{\mu, \gamma}(a ; b)$ we shall require the following estimates for the gamma function and the Riemann zeta function $\zeta(s)$. For real $\sigma$ and $t$, we have the estimates

$$
\Gamma(\sigma \pm i t)=O\left(t^{\sigma-\frac{1}{2}} e^{-\frac{1}{2} \pi t}\right), \quad|\zeta(\sigma \pm i t)|=O\left(t^{\omega(\sigma)} \log ^{\alpha} t\right) \quad(t \rightarrow+\infty)
$$

\footnotetext{
${ }^{1}$ The restriction $\gamma>-1$ is imposed for convenience to avoid the possible formation of a double pole in (2.3) below at $s=1$ when $\gamma$ equals a negative integer.
} 
where $\omega(\sigma)=0(\sigma>1), \frac{1}{2}-\frac{1}{2} \sigma(0 \leq \sigma \leq 1), \frac{1}{2}-\sigma(\sigma<0)$ and $\alpha=1$ $(0 \leq \sigma \leq 1), \alpha=0$ otherwise [3, p. 25], [11, p. 95]. The zeta function $\zeta(s)$ has a simple pole of unit residue at $s=1$ and the evaluations for positive integer $k$

$$
\begin{gathered}
\zeta(0)=-\frac{1}{2}, \quad \zeta(-2 k)=0, \quad \zeta(1-2 k)=-\frac{B_{2 k}}{2 k}, \\
B_{0}=1, \quad B_{2}=\frac{1}{6}, B_{4}=-\frac{1}{30}, B_{6}=\frac{1}{42}, \ldots,
\end{gathered}
$$

where $B_{k}$ are the Bernoulli numbers. Finally, we have the well-known functional relation satisfied by $\zeta(s)$ given by

$$
\zeta(s)=2^{s} \pi^{s-1} \zeta(1-s) \Gamma(1-s) \sin \frac{1}{2} \pi s .
$$

\section{Asymptotic expansion for $|a| \rightarrow \infty$ in $|\arg a|<\frac{1}{2} \pi$}

We first consider the generalised Mathieu series $S_{\mu, \gamma}(a ; b)$ defined in (1.3) by

$$
S_{\mu, \gamma}(a ; b)=a^{-\delta} e^{-b} \sum_{n=1}^{\infty} h(n / a), \quad h(x):=\frac{x^{\gamma}}{\left(1+x^{2}\right)^{\mu}} \exp \left[\frac{b x^{2}}{1+x^{2}}\right] .
$$

We shall employ a Mellin transform approach as discussed in [9, Section 4.1.1]. The Mellin transform of $h(x)$ is $H(s)=\int_{0}^{\infty} x^{s-1} h(x) d x$, where

$$
\begin{aligned}
H(s) & =\int_{0}^{\infty} \frac{x^{\gamma+s-1}}{\left(1+x^{2}\right)^{\mu}} \exp \left[\frac{b x^{2}}{1+x^{2}}\right] d x=\frac{1}{2} \sum_{r=0}^{\infty} \frac{b^{r}}{r !} \int_{0}^{\infty} \frac{\tau^{\frac{1}{2} \gamma+\frac{1}{2} s+r-1}}{(1+\tau)^{\mu+r}} d \tau \\
& =\frac{1}{2} \sum_{r=0}^{\infty} \frac{b^{r}}{r !} \frac{\Gamma\left(\frac{1}{2} \gamma+\frac{1}{2} s+r\right) \Gamma\left(\mu-\frac{1}{2} \gamma-\frac{1}{2} s\right)}{\Gamma(\mu+r)} \\
& =\frac{\Gamma\left(\frac{1}{2} \gamma+\frac{1}{2} s\right) \Gamma\left(\mu-\frac{1}{2} \gamma-\frac{1}{2}\right)}{2 \Gamma(\mu)} F(s), \quad F(s):={ }_{1} F_{1}\left(\frac{1}{2}(\gamma+s) ; \mu ; b\right)
\end{aligned}
$$

in the strip $-\gamma<\Re(s)<\delta$. The evaluation of the integral has been carried out by the beta function and ${ }_{1} F_{1}$ denotes the confluent hypergeometric function. Using the Mellin inversion theorem (see [9, p. 118]) we find

$$
\sum_{n=1}^{\infty} h(n / a)=\frac{1}{2 \pi i} \sum_{n=1}^{\infty} \int_{c-\infty i}^{c+\infty i} H(s)(n / a)^{-s} d s=\frac{1}{2 \pi i} \int_{c-\infty i}^{c+\infty i} H(s) \zeta(s) a^{s} d s,
$$

where $1<c<\delta$ and $\zeta(s)$ is the Riemann zeta function. The inversion of the order of summation and integration is justified by absolute convergence provided $1<c<\delta$. Then, from (2.1),

$$
S_{\mu, \gamma}(a ; b)=\frac{a^{-\delta} e^{-b}}{2 \Gamma(\mu)} \frac{1}{2 \pi i} \int_{c-\infty i}^{c+\infty i} \Gamma\left(\frac{1}{2} \gamma+\frac{1}{2} s\right) \Gamma\left(\mu-\frac{1}{2} \gamma-\frac{1}{2} s\right) F(s) \zeta(s) a^{s} d s,
$$


where $1<c<\delta$.

From [4, Theorem 2.3] we have for finite values of $\mu$ and $b \neq 0$

$$
F(s)=\frac{\Gamma(\mu)}{\sqrt{ } \pi} e^{b / 2}(\kappa b)^{\frac{1}{4}-\frac{1}{2} \mu} \cos \left(2 \sqrt{\kappa b}-\frac{1}{2} \pi b+\frac{1}{4} \pi\right)\left\{1+O\left(|\kappa|^{-\frac{1}{2}}\right)\right\}
$$

as $|\kappa| \rightarrow \infty$ in $-\pi<\arg \kappa \leq \pi$, where $\kappa:=\frac{1}{2}(\mu-\gamma-s)$. It then follows that with $s=\sigma+i t$, where $\sigma$ and $t$ are real,

$$
F(s)=O\left(|t|^{\frac{1}{4}-\frac{1}{2} \mu} \exp [\sqrt{|b t|}]\right), \quad t \rightarrow \pm \infty .
$$

From the estimates in (1.4), the integral in (2.3) then defines $S_{\mu, \gamma}(a ; b)$ for complex $a$ in the sector $|\arg a|<\frac{1}{2} \pi$.

Since ${ }_{1} F_{1}(A ; B ; z)$ is entire in the parameter $A[7$, p. 322], the integrand in (2.3) has a simple pole at $s=1$ and infinite strings of simple poles at $s=-\gamma-2 k$ and $s=2 \mu-\gamma+2 k, k=0,1,2, \ldots$. Let $N$ be a positive integer and let $c^{\prime}=2 N+\gamma-1$. We consider the integral in (2.3) taken round the rectangular contour with vertices at $c \pm i T$ and $-c^{\prime} \pm i T$, where $T>0$, so that the side in $\Re(s)<0$ parallel to the imaginary $s$-axis passes midway between the poles at $s=-2(N-1)-\gamma$ and $s=-2 N-\gamma$. The contribution from the upper and lower sides of the rectangle $s=\sigma \pm i T,-c^{\prime} \leq \sigma \leq c$, vanishes as $T \rightarrow \infty$ provided $|\arg a|<\frac{1}{2} \pi$, since, from (1.4) and (2.4), the modulus of the integrand is controlled by $O\left(T^{\omega(\sigma)+\frac{1}{2} \mu-\frac{3}{4}} \log T \exp [-\Delta T+\sqrt{|b| T}]\right)$, where $\Delta=\frac{1}{2} \pi-|\arg a|$. Evaluation of the residues ${ }^{2}$ at $s=1$ and $s=-\gamma-2 k$ $(0 \leq k \leq N-1)$ then yields

$$
\begin{aligned}
S_{\mu, \gamma}(a ; b)=\frac{\Gamma\left(\frac{1}{2} \gamma+\frac{1}{2}\right) \Gamma\left(\mu-\frac{1}{2} \gamma-\frac{1}{2}\right) F(1)}{2 e^{b} \Gamma(\mu) a^{2 \mu-\gamma-1}} & \\
& +\frac{e^{-b}}{\Gamma(\mu)} \sum_{k=0}^{N-1} \frac{(-1)^{k} \Gamma(\mu+k)}{k ! a^{2(k+\mu)}} \zeta(-\gamma-2 k) C_{k}+R_{N}(a),
\end{aligned}
$$

where the coefficients $C_{k}$ are defined by

$$
C_{k}={ }_{1} F_{1}(-k ; \mu ; b) \quad k=0,1,2, \ldots .
$$

The $C_{k}$ are consequently polynomials in $b$ of degree $k$ and

$$
C_{0}=1, \quad C_{1}=1-\frac{b}{\mu}, \quad C_{2}=1-\frac{2 b}{\mu}+\frac{b^{2}}{\mu(\mu+1)}, \ldots .
$$

The remainder integral $R_{N}(a)$ is given by

$$
\begin{aligned}
R_{N}(a) & =\frac{a^{-\delta} e^{-b}}{2 \Gamma(\mu)} \frac{1}{2 \pi i} \int_{-c^{\prime}-\infty i}^{-c^{\prime}+\infty i} \Gamma\left(\frac{1}{2} s+\frac{1}{2} \gamma\right) \Gamma\left(\mu-\frac{1}{2} \gamma-\frac{1}{2} s\right) F(s) \zeta(s) a^{s} d s \\
& =\frac{a^{-\delta} e^{-b}}{4 \Gamma(\mu)} \frac{1}{2 \pi i} \int_{-c^{\prime}-\infty i}^{-c^{\prime}+\infty i} \frac{\Gamma\left(\frac{1}{2} s+\frac{1}{2} \gamma\right) \Gamma\left(\mu-\frac{1}{2} \gamma-\frac{1}{2} s\right)}{\Gamma(s) \cos \frac{1}{2} \pi s(2 \pi a)^{-s}} F(s) \zeta(1-s) d s
\end{aligned}
$$

\footnotetext{
${ }^{2}$ The residue of $\Gamma(s)$ at $s=-k$ is $(-1)^{k} / k$ !.
} 
Here we have used the functional relation in (1.6) and the reflection formula for the gamma function. If we set $s=-c^{\prime}+i t$ and use the fact that $|\zeta(\sigma+i t)| \leq$ $\zeta(\sigma)$ when $\sigma>1$, we find

$$
\left|R_{N}(a)\right| \leq \frac{(2 \pi)^{-2 N-\gamma} \zeta(2 N+\gamma)}{4 e^{b} \Gamma(\mu)|a|^{2 N+2 \mu-1}} \int_{-\infty}^{\infty} g(t)\left|F\left(-c^{\prime}+i t\right)\right| e^{\theta t-\frac{1}{2} \pi|t|} d t,
$$

where

$$
g(t)=\left|\frac{\Gamma\left(-N+\frac{1}{2}+\frac{1}{2} i t\right) \Gamma\left(\mu+N-\frac{1}{2}-\frac{1}{2} i t\right) e^{\frac{1}{2} \pi|t|}}{\Gamma\left(-c^{\prime}+i t\right) \cos \frac{1}{2} \pi\left(-c^{\prime}+i t\right)}\right|
$$

and $\theta=\arg a$. Since $g(t)=O\left(|t|^{\mu+c^{\prime}-\frac{1}{2}}\right)$ as $t \rightarrow \pm \infty$, by the first estimate in (1.4), it follows from (2.4) that the above integral is independent of $|a|$ and converges when $|\arg a|<\frac{1}{2} \pi$. Hence $R_{N}(a)=O\left(|a|^{-2 N-2 \mu+1}\right)$ and we consequently obtain the expansion:

Theorem 1. With the conditions on the parameters $\mu, \gamma$ given in (1.4), we have the asymptotic expansion

$$
\begin{aligned}
S_{\mu, \gamma}(a ; b) \sim & \frac{\Gamma\left(\frac{1}{2} \gamma+\frac{1}{2}\right) \Gamma\left(\mu-\frac{1}{2} \gamma-\frac{1}{2}\right) F(1)}{2 e^{b} \Gamma(\mu) a^{2 \mu-\gamma-1}} \\
& +\frac{e^{-b}}{\Gamma(\mu)} \sum_{k=0}^{\infty} \frac{(-1)^{k} \Gamma(\mu+k)}{k ! a^{2(k+\mu)}} \zeta(-\gamma-2 k) C_{k}
\end{aligned}
$$

for $|a| \rightarrow \infty$ in the sector $|\arg a|<\frac{1}{2} \pi$. The quantity $F(1)$ is defined in (2.2) and the coefficients $C_{k}$ in (2.5).

The expansion of the alternating series $\tilde{S}_{\mu, \gamma}(a ; b)$ can be deduced from that for $S_{\mu, \gamma}(a ; b)$ in $(2.6)$ by observing that for $\delta>1$

$$
\tilde{S}_{\mu, \gamma}(a ; b)=S_{\mu, \gamma}(a ; b)-2^{1-\delta} S_{\mu, \gamma}\left(\frac{1}{2} a ; b\right) .
$$

Then, from (2.6), we obtain the following result.

Theorem 2. With the conditions on the parameters $\mu, \gamma$ given in (1.4), we have the asymptotic expansion when $\delta>1$

$$
\tilde{S}_{\mu, \gamma}(a ; b) \sim \frac{e^{-b}}{\Gamma(\mu)} \sum_{k=1}^{\infty} \frac{(-1)^{k-1} \Gamma(\mu+k)}{k ! a^{2 k+2 \mu}}\left(2^{2 k+\gamma+1}-1\right) \zeta(-\gamma-2 k) C_{k}
$$

for $|a| \rightarrow \infty$ in the sector $|\arg a|<\frac{1}{2} \pi$, where the coefficients $C_{k}$ are specified in (2.5).

In the case of the standard Mathieu series in (1.1) and (1.2), with $\mu=2$, $\gamma=1, b=0$, we find from (2.6) and (2.7) that

$$
S_{2}(a) \sim \frac{1}{2} \sum_{k=0}^{\infty} \frac{(-1)^{k} B_{2 k}}{a^{2 k+2}}, \quad \tilde{S}_{2}(a) \sim \frac{1}{2} \sum_{k=0}^{\infty} \frac{(-1)^{k}}{a^{2 k+2}}\left(2^{2 k}-1\right) B_{2 k}
$$


as $|a| \rightarrow \infty$ in $|\arg a|<\frac{1}{2} \pi$, which agree with previous results ${ }^{3}$ for the case $a \rightarrow+\infty[1,10]$. In these last expansions we have replaced $\zeta(-1-2 k)$ by its representation in terms of the Bernoulli numbers by (1.5) and made the change of summation index $k \rightarrow k-1$.

The above procedure can be extended without difficulty to the more general functional series

$$
S_{\mu, \gamma, \lambda}(a ; b)=\sum_{n=1}^{\infty} \frac{n^{\gamma}}{\left(n^{\lambda}+a^{\lambda}\right)^{\mu}} \exp \left[\frac{-a^{\lambda} b}{n^{\lambda}+a^{\lambda}}\right] \quad(\mu>0, \lambda>0, \gamma>-1, b \in \mathbf{R})
$$

and its alternating variant $\tilde{S}_{\mu, \gamma, \lambda}(a ; b)$, where it is supposed that $\delta:=\lambda \mu-\gamma>1$ for absolute convergence of both series. For complex $a$ it is sufficient to confine our attention to the sector $|\arg a| \leq \pi / \lambda$; if $\lambda$ is non-integer then a branchpoint structure is introduced. Then, for $1<c<\delta$,

$$
\begin{gathered}
S_{\mu, \gamma, \lambda}(a ; b)=\frac{a^{-\delta} e^{-b}}{\lambda \Gamma(\mu)} \frac{1}{2 \pi i} \int_{c-\infty i}^{c+\infty i} \Gamma\left(\frac{\gamma+s}{\lambda}\right) \Gamma\left(\mu-\frac{\gamma+s}{\lambda}\right) F_{\lambda}(s) \zeta(s) a^{s} d s \\
F_{\lambda}(s):={ }_{1} F_{1}\left(\frac{\gamma+s}{\lambda} ; \mu ; b\right)
\end{gathered}
$$

and, omitting the details, we find the following asymptotic expansions.

Theorem 3. With the parameters defined in (2.8), we have the asymptotic expansions when $\delta=\lambda \mu-\gamma>1$

$S_{\mu, \gamma, \lambda}(a ; b) \sim \frac{\Gamma\left(\frac{\gamma+1}{\lambda}\right) \Gamma\left(\mu-\frac{\gamma+1}{\lambda}\right) F_{\lambda}(1)}{\lambda e^{b} \Gamma(\mu) a^{\lambda \mu-\gamma-1}}+\frac{e^{-b}}{\Gamma(\mu)} \sum_{k=0}^{\infty} \frac{(-1)^{k} \Gamma(\mu+k)}{k ! a^{\lambda(k+\mu)}} \zeta(-\gamma-\lambda k) C_{k}$

and

$$
\tilde{S}_{\mu, \gamma, \lambda}(a ; b) \sim \frac{e^{-b}}{\Gamma(\mu)} \sum_{k=0}^{\infty} \frac{(-1)^{k-1} \Gamma(\mu+k)}{k ! a^{\lambda(k+\mu)}}\left(2^{\lambda k+\gamma+1}-1\right) \zeta(-\gamma-\lambda k) C_{k}
$$

for $|a| \rightarrow \infty$ in the sector $|\arg a|<\pi / \lambda$. The quantity $F_{\lambda}(1)$ and the coefficients $C_{k}$ are defined in (2.9) and (2.5).

In the case $b=0$ the expansions in (2.10) and (2.11) agree with those derived in [12] for $a \rightarrow+\infty$. We note that when $\lambda=2, \gamma=1$ the expansions (2.10) and (2.11) reduce to (2.6) and (2.7), respectively. Also, in the case $\lambda=1, \mu=1,-1<\gamma<0$ and $b=0$ the expansion of $S_{1, \gamma}(a ; 0)$ agrees with that given in $[9,(4.2 .3)]$ with $\alpha=\gamma+1$, viz.

$$
\sum_{n=1}^{\infty} \frac{n^{\alpha-1}}{n+a} \sim \frac{\pi a^{\alpha-1}}{\sin \pi \alpha}+\sum_{k=0}^{\infty} \frac{(-1)^{k}}{a^{k+1}} \zeta(1-\alpha-k) \quad(0<\alpha<1)
$$

\footnotetext{
${ }^{3}$ There is a misprint in (3.6) of [10]; a factor $(-1)^{j}$ is missing from the sum on the left-hand side and the leading term should be $1 /\left(4 r^{4}\right)$.
} 
as $|a| \rightarrow \infty$ in $|\arg a|<\pi$.

\section{Numerical results}

We present in Table 1 some numerical results to illustrate the accuracy of the expansions $(2.6),(2.7),(2.10)$ and (2.11) for different parameters when $a=10 e^{i \theta}$ with $|\theta|<\pi / \lambda$. The values shown give the absolute relative error in the computation of the different generalised Mathieu series compared with high-precision evaluation of the infinite series.

\begin{tabular}{|c|c|c|}
\hline $\begin{array}{c}b=0 \\
\mu=1.5, \lambda=2, \gamma=1 \\
\theta\end{array}$ & $\begin{array}{c}c=-0.5 \\
\mu=3, \lambda=1.5, \gamma=1 \\
\theta \quad \text { Error }\end{array}$ & 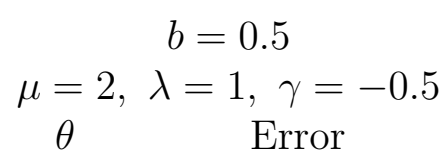 \\
\hline $\begin{array}{ll}0 & 3.713 \times 10^{-11} \\
0.25 \pi & 3.703 \times 10^{-11} \\
0.40 \pi & 4.613 \times 10^{-6}\end{array}$ & $\begin{array}{ll}0 & 9.105 \times 10^{-11} \\
0.30 \pi & 8.925 \times 10^{-11} \\
0.50 \pi & 5.662 \times 10^{-9}\end{array}$ & $\begin{array}{ll}0 & 4.980 \times 10^{-6} \\
0.50 \pi & 4.473 \times 10^{-6} \\
0.75 \pi & 4.019 \times 10^{-6}\end{array}$ \\
\hline \begin{tabular}{c}
\multicolumn{2}{c}{$b=0$} \\
$\mu=1.5, \lambda=2, \gamma=1$ \\
$\theta \quad$ Error
\end{tabular} & \begin{tabular}{cc}
\multicolumn{2}{c}{$b=-0.5$} \\
$\mu=3, \lambda=1.5, \gamma=1$ \\
$\theta \quad$ Error
\end{tabular} & \begin{tabular}{c}
\multicolumn{2}{c}{$b=0.5$} \\
$\mu=2, \lambda=1, \gamma=-0.5$ \\
$\theta \quad$ Error
\end{tabular} \\
\hline $\begin{array}{l}1.924 \times 10^{-9} \\
1.337 \times 10^{-5} \\
5.689 \times 10^{-4}\end{array}$ & $\begin{array}{l}1.030 \times 10^{-7} \\
9.956 \times 10^{-7} \\
1.635 \times 10^{-4}\end{array}$ & $\begin{array}{l}1.551 \times 10^{-9} \\
1.904 \times 10^{-9} \\
2.572 \times 10^{-7}\end{array}$ \\
\hline
\end{tabular}

Table 1: The absolute relative error in the computation of $S_{\mu, \gamma, \lambda}(a ; b)$ (upper table) and $\tilde{S}_{\mu, \gamma, \lambda}(a ; b)$ (lower table) using the asymptotic expansions (2.10) and (2.11) for different parameters when $a=10 \exp (i \theta)$. The truncation index in each case is $k=10$.

The value of $\zeta(-\gamma-\lambda k)$ appearing in these expansions can be evaluated by means of (1.6) and, in the case of integer values of $\gamma$ and $\lambda$ by means of (1.5). The optimal truncation index of a (divergent) asymptotic expansion corresponds to truncation at, or near, the least term in magnitude. From (1.6) and the well-known fact that $\Gamma(k+a) / \Gamma(k+b) \sim k^{a-b}$ for $k \rightarrow+\infty$, the optimal truncation index $k_{0}$ for the asymptotic series in $(2.10)$ is found to be

$$
k_{0} \simeq \frac{2 \pi|a|}{\lambda}
$$

with that for the series (2.11) being reduced by a factor of 2 . It is then seen that with $|a|=10$, the truncation index $k=10$ employed in Table 1 is sub-optimal in each case. 


\section{References}

[1] A. Elbert, Asymptotic expansion and continued fraction for Mathieu's series, Period. Math. Hungar. 13 (1982) 1-8.

[2] O. Emersleben, Über die Reihe $\sum_{k=1}^{\infty} k /\left(k^{2}+c^{2}\right)^{2}$, Math. Ann. 125 (1952) $165-171$.

[3] A. Ivić, The Riemann Zeta Function, Wiley, New York, 1985.

[4] J. L. López, Asymptotic expansions of the Whittaker functions for large order parameters, Methods Appl. Anal. 6(2) (1999) 249-256.

[5] E. L. Mathieu, Traité de Physique Mathématique. VI-VII: Théorie de l'Élasticité des Corps Solides (Part 2), Gauthier-Villars, Paris, 1890.

[6] G. V. Milovanović and T. K. Pogány, New integral forms of generalized Mathieu series and related applications, Appl. Anal. Discrete Math. 7 (2013) 180-192.

[7] F. W. J. Olver, D. W. Lozier, R. F. Boisvert and C. W. Clark (eds.), NIST Handbook of Mathematical Functions, Cambridge University Press, Cambridge, 2010.

[8] R. B. Paris, On the growth of perturbations of the exponential series, Math. Balkanica 21 (2007) 183-199.

[9] R. B. Paris and D. Kaminski, Asymptotics and Mellin-Barnes Integrals, Cambridge University Press, Cambridge, 2001.

[10] T. K. Pogány, H. M. Srivastava and Z. Tomovski, Some families of Mathieu a-series and alternating Mathieu a-series, Appl. Math. Comp. 173 (2006) 69-108.

[11] E. C. Titchmarsh, The Theory of the Riemann Zeta-Function. Revised by D. R. Heath-Brown, Oxford University Press, Oxford, 1986.

[12] V. P. Zastavnyi, Asymptotic expansions of several series and their application, Ukrainian Math. Bull. 6 (2009) 549-569.

\section{Received: September 3, 2013}

